\title{
CyberKnife boost for patients with cervical cancer unable to undergo brachytherapy
}

\section{Jonathan Andrew Haas ${ }^{1 *}$, Matthew R. Witten ${ }^{2}$, Owen Clancey ${ }^{2}$, Karen Episcopia ${ }^{2}$, Diane Accordino ${ }^{1}$ and Eva Chalas $^{3}$}

1 Division of Radiation Oncology, Winthrop-University Hospital, Mineola, NY, USA

${ }^{2}$ Division of Medical Physics, Winthrop-University Hospital, Mineola, NY, USA

${ }^{3}$ Division of Gynecologic Oncology, Winthrop-University Hospital, Mineola, NY, USA

\section{Edited by:}

Brian Timothy Collins, Georgetown Hospital, USA

Reviewed by:

Sean Collins, Georgetown University Hospital, USA

Brian Timothy Collins, Georgetown Hospital, USA

\section{${ }^{*}$ Correspondence:}

Jonathan Andrew Haas, Division of

Radiation Oncology,

Winthrop-University Hospital, 264 Old

Country Road, Mineola, NY 11501,

USA

e-mail: jhaas@winthrop.org
Standard radiation therapy for patients undergoing primary chemosensitized radiation for carcinomas of the cervix usually consists of external beam radiation followed by an intracavitary brachytherapy boost. On occasion, the brachytherapy boost cannot be performed due to unfavorable anatomy or because of coexisting medical conditions. We examined the safety and efficacy of using CyberKnife stereotactic body radiotherapy (SBRT) as a boost to the cervix after external beam radiation in those patients unable to have brachytherapy to give a more effective dose to the cervix than with conventional external beam radiation alone. Six consecutive patients with anatomic or medical conditions precluding a tandem and ovoid boost were treated with combined external beam radiation and CyberKnife boost to the cervix. Five patients received $45 \mathrm{~Gy}$ to the pelvis with serial intensity-modulated radiation therapy boost to the uterus and cervix to a dose of $61.2 \mathrm{~Gy}$. These five patients received an SBRT boost to the cervix to a dose of $20 \mathrm{~Gy}$ in five fractions of $4 \mathrm{~Gy}$ each. One patient was treated to the pelvis to a dose of $45 \mathrm{~Gy}$ with an external beam boost to the uterus and cervix to a dose of $50.4 \mathrm{~Gy}$. This patient received an SBRT boost to the cervix to a dose of $19.5 \mathrm{~Gy}$ in three fractions of $6.5 \mathrm{~Gy}$. Five percent volumes of the bladder and rectum were kept to $\leq 75 \mathrm{~Gy}$ in all patients (i.e., $\mathrm{V} 75 \mathrm{~Gy} \leq 5 \%$ ). All of the patients remain locally controlled with no evidence of disease following treatment. Grade 1 diarrhea occurred in $4 / 6$ patients during the conventional external beam radiation. There has been no grade 3 or 4 rectal or bladder toxicity. There were no toxicities observed following SBRT boost. At a median follow-up of 14 months, CyberKnife radiosurgical boost is well tolerated and efficacious in providing a boost to patients with cervix cancer who are unable to undergo brachytherapy boost. Further follow-up is required to see if these results remain durable.

Keywords: cervix, SBRT, brachytherapy, CyberKnife

\section{INTRODUCTION}

Standard radiation therapy for cervical carcinoma patients undergoing primary chemosensitized radiation therapy usually consists of external beam therapy followed by an intracavitary brachytherapy boost (Eifel et al., 2004). Using this approach, the brachytherapy serves to provide a tumorical dose to the cervix while limiting the dose to surrounding anatomy, such as the bladder and rectum, which have a lower dose tolerance. Typically, external beam radiation delivers a dose of approximately $45 \mathrm{~Gy}$ to encompass the primary tumor and regional pelvic lymph nodes. The brachytherapy boost results in a total dose ranging from 70 to $95 \mathrm{~Gy}$ to the primary tumor (assuming an alpha/beta ratio of $10 \mathrm{~Gy}$ ), depending on tumor stage and anatomy.

In certain circumstances, the brachytherapy boost may not be feasible due to coexisting medical conditions, unfavorable anatomy, or patient refusal to undergo the procedure. In these cases, a higher dose of external beam radiation (EBRT) may be given, but the total dose delivered is usually less than when a brachytherapy procedure is performed. Predictably, the results when brachytherapy is not performed are inferior. For instance, Barraclough et al. delivered a total dose of 54-70 Gy through the addition of an EBRT boost to patients unable to receive the brachytherapy boost. The majority of patients developed a central recurrence in less than 5 years and had a 5year overall survival rate of $49.3 \%$ (Barraclough et al., 2008). This compares unfavorably to combined external beam therapy with a brachytherapy boost where 5-year local control and survival are in the $60-70 \%$ range (Rose et al., 1999; Eifel et al., 2004).

Brachytherapy takes advantage of the inverse square law in that high doses of radiation are given to the target (cervix in this case) and low doses are given to the normal anatomy by moving them out of the way with packing material exploiting the rapid dose falloff and inhomogeneous dose distribution seen with brachytherapy. Stereotactic body radiotherapy (SBRT) provides a potential alternative method to boost the cervix in those cases where brachytherapy is not performed. SBRT emulates brachytherapy by having multiple non-coplanar beams intersecting at the target (cervix) delivering a high therapeutic dose while minimizing beam traversal through normal anatomy reducing dose to these areas. By 
prescribing to a specific isodose line (i.e., the dose line that covers the volume of interest), the tumor receives an inhomogeneous dose similar to that delivered with brachytherapy. Technological advances using the CyberKnife (Accuray Incorporated, Sunnyvale, CA, USA), a robotic radiation delivery system, allow more precise targeting and delivery of radiation to the cervix while sparing normal anatomy compared to conventional radiation. This potentially allows dose escalation to the cervix to a dose comparable to the brachytherapy boost while respecting the normal tissue tolerance of the bladder and rectum. The CyberKnife's use of a large number of small radiation beams also allows delivery of an inhomogeneous dose distribution similar to that of brachytherapy (Fuller et al., 2008).

Another important aspect of CyberKnife delivered SBRT to the cervix is motion tracking. The cervix is not a fixed pelvic organ, rather one that is subject to movement during treatment. For example, a study from the University of California at San Diego showed that the cervix can move as much as $18 \mathrm{~mm}$ during intensity-modulated radiation therapy (IMRT) treatment (Haripotepornkul et al., 2011); other studies of cervical motion have shown similar movement (Hombaiah et al., 2006; Taylor and Powell, 2008). Given the large movement possible, continuous tracking of the cervix during treatment is imperative in ensuring proper delivery of dose to the tumor particularly when giving larger than conventional daily doses of radiation as is the case with the SBRT. Lastly, since SBRT is given in a week, the total treatment time frame is comparable to external beam therapy with a brachytherapy boost thus limiting the deleterious effect observed in prolonged treatments extending beyond 7 weeks (Fyles et al., 1992; Girinsky et al., 1993; Lanciano et al., 1993; Perez et al., 1995; Petereit et al., 1995).

In this report, we present preliminary local control results on the treatment of six patients with cervical cancer who did not have a brachytherapy boost and were treated with an SBRT boost resulting in a total dose of $77-85$ Gy to the cervix.

\section{MATERIALS AND METHODS}

This is a retrospective chart review of cervical cancer patients treated with combined external beam radiation and SBRT boost to the cervix at Winthrop-University Hospital from 3/2009 to 8/2011. All patients gave informed consent for the treatment. All patients received a series of conventionally fractionated radiation therapy followed by an SBRT dose. One of two dose schemes was used. Early in our program of treating cervical cancer patients with an SBRT boost the conventionally fractionated treatment consisted of a 45 Gy dose to the pelvis using $15 \mathrm{MV}$ photons followed by an IMRT boost to the cervix and uterus to a total delivered conventionally fractionated radiation therapy dose of $50.4 \mathrm{~Gy}$. Subsequently, we modified our treatment so that the conventionally fractionated treatment began with a dose of $45 \mathrm{~Gy}$ to the pelvis using $15 \mathrm{MV}$ photons followed by two IMRT boosts, one to the uterus and cervix that increased the delivered dose to 50.4 Gy and a second IMRT boost to the cervix alone resulting in a total delivered conventionally fractionated radiation therapy dose of $61.2 \mathrm{~Gy}$. The bladder and rectum dose constraints required no more than $5 \%$ of their volume to receive $70 \mathrm{~Gy}$ (i.e., V70 Gy $\leq 5 \%$ ). However, in cases where the tumor's anatomical location necessitated a high dose to the bladder and/or rectum a point dose of up to 75 Gy was allowed.

Following conventionally fractionated radiation therapy, patients had three to four gold fiducial markers placed into the cervix and upper vagina. Fiducial placement began with a lidocaine gel and a betadine prep and proceeded under direct visualization in the lithotomy position. The fiducials were placed into the cervix at the 3 and 9 o'clock position and superiorly into the vaginal fornices in an orientation to prevent overlap in the plane of the $\mathrm{X}$-ray imaging. Treatment planning CT scans at a slice thickness of $1.25 \mathrm{~mm}$ and an MRI scan using a slice thickness of 1-2 mm were performed 1 week after fiducial placement. All pretreatment imaging was performed with the patient in the same position used for SBRT delivery. The pulse sequence used for MRI acquisition was gradient echo which maximized the signal void attributable to the fiducials and allowed for clear visualization of the fiducials in the MR image. This allowed for the fusion of the MR and CT data sets using the fiducials. The gross tumor volume (GTV) was contoured by the attending radiation oncologist using both CT and MRI images to accurately delineate the cervical anatomy and to define the interface between the cervix and anterior wall of the rectum.

All patients received SBRT boost using the CyberKnife system (Accuray Incorporated, Sunnyvale, CA, USA) which consists of a 6-MV linear accelerator mounted on a robotic arm. Using this system two orthogonal kilovoltage X-ray imagers provide realtime image guidance and automatic correction for movement of the cervix throughout the treatment. There were typically 150 200 non-coplanar beams delivered during each treatment session. Target tracking and patient positioning were accomplished by registering the location of the fiducial markers in the real-time images to their planning CT location. Imaging was performed approximately once per minute. The robotic delivery system automatically changes the linear accelerator's position to correct for both rotational and translational movement of the patient and cervix during treatment. The total clinical accuracy for treatment is less than $1 \mathrm{~mm}$ (Kilby et al., 2010). Treatments were delivered on three or five consecutive days. Patients had a bowel prep including Dulcolax (Boehringer, Germany) and a fleet enema on the morning of each treatment. Additionally, patients received $1500 \mathrm{mg}$ of amifostine (MedImmune, LLC, Gaithersburg, MD, USA) mixed in saline as a rectal suppository at least $15-20 \mathrm{~min}$ prior to each treatment as a radioprotectant.

For the lower conventionally fractionated dose (50.4 Gy) the SBRT boost to the cervix was $19.5 \mathrm{~Gy}$ in three fractions of $6.5 \mathrm{~Gy}$ each. For later patients who received a conventionally fractionated dose of $61.2 \mathrm{~Gy}$ the SBRT boost to the cervix was $20 \mathrm{~Gy}$ in five fractions of $4 \mathrm{~Gy}$ each. In all cases, margins were $5 \mathrm{~mm}$ laterally, inferiorly and superiorly around the CTV. The margins were $3 \mathrm{~mm}$ anteriorly and posteriorly to limit dose to the bladder and rectum. Four of the six patients received systemic chemotherapy during their radiation consisting of cisplatin at a dose of $40 \mathrm{mg} / \mathrm{m}^{2}$.

Patients were followed at 3 weeks after treatment and every 3 months thereafter. Follow-up assessments were based on physical examination by the radiation oncologist and treating gynecologic oncologist. Toxicities were scored based on radiation therapy oncology group (RTOG) rectal and urinary toxicity criteria. 


\section{RESULTS}

Six consecutive cervical cancer patients were treated with combined external beam radiation and SBRT boost to the cervix at Winthrop-University Hospital from 3/2009 to 8/2011. The median patient age was 80 years (range, 71-94 years). One patient refused brachytherapy; all other patients were unable to receive a tandem and ovoid brachytherapy boost because of either anatomic $(n=3)$ or medical $(n=2)$ conditions. The first patient treated received a conventionally fractionated total dose of $50.4 \mathrm{~Gy}$ followed by an SBRT cervix boost of $19.5 \mathrm{~Gy}$ delivered in three fractions. The five subsequent patients received a conventionally fractionated total dose of 61.2 Gy followed by an SBRT cervix boost of 20 Gy delivered in four fractions. Five percent volumes of the bladder and rectum were kept to $\leq 70 \mathrm{~Gy}$ (i.e., V75 Gy $\leq 5 \%$ ) with the exception of maximal post doses up to $75 \mathrm{~Gy}$ when necessary based on tumor location (Table 2) Figure 1 shows a sample treatment plan and dose volume histogram for a patient receiving an SBRT boost of $20 \mathrm{~Gy}$. Observed cervix motion during treatment consisted of drift in the anterior-posterior or superior-inferior axes; sporadic movement in the anterior-posterior, superior-inferior, or left-right axes; or a combination of the two. This movement was typically on the order of $10 \mathrm{~mm}$. Complete details on patient characteristics and treatment parameters are summarized in Table $\mathbf{1 .}$ In addition, Table 2 summarizes the maximal rectal and bladder doses for each patient.

All patients tolerated the treatment well with no grade 3 or higher urinary or rectal toxicities. Grade 1/2 urinary and bowel toxicities occurred in four patients following conventional external beam radiation. All of these symptoms resolved by the time of SBRT boost. At a median follow-up of 14 months (range, 128 months) from completion of the SBRT boost there have been no additional RTOG toxicities. In addition, for the five patients with a minimum of 12 months follow-up all (100\%) remain locally and distantly controlled with no evidence of disease.

\section{DISCUSSION}

This report demonstrates the feasibility of using robotic SBRT as an alternative to brachytherapy in cervical cancer patients unable to undergo brachytherapy. The motivation for this series stems from the markedly inferior reported outcomes for treatment of such patients with a conventionally fractionated radiation boost compared to brachytherapy. Specifically, Barraclough et al. (2008) report on the treatment of 44 patients with a conventional external beam boost to a total dose of 54-70 Gy when intracavity therapy could not be performed. At a median 2.3 years follow-up, recurrent disease was seen in $48 \%$ of patients with a median time to recurrence of 2.3 years. In addition, they observed late grade 3 toxicity in $2 \%$ of patients and late grade 1 and 2 bowel and bladder toxicities in $41 \%$ of patients (Barraclough et al., 2008). The poor outcomes in these studies are likely explained by the low doses given to the paracervical region in order to respect tissue tolerance of the surrounding anatomy such as the bladder and rectum. Indeed, most institutions do not exceed 75-80 Gy (combined external beam and LDR brachytherapy dose) to the International Commission on Radiological Units (ICRU) and Measurements bladder reference point and 70-75 Gy to the rectal reference point (Fletcher and Hamberger, 1980; Eifel et al., 2004).

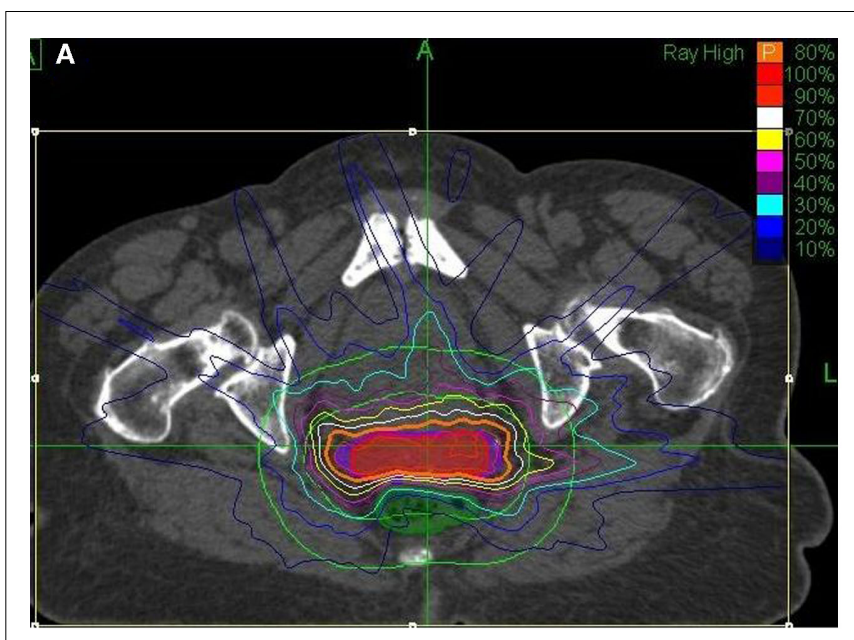

B
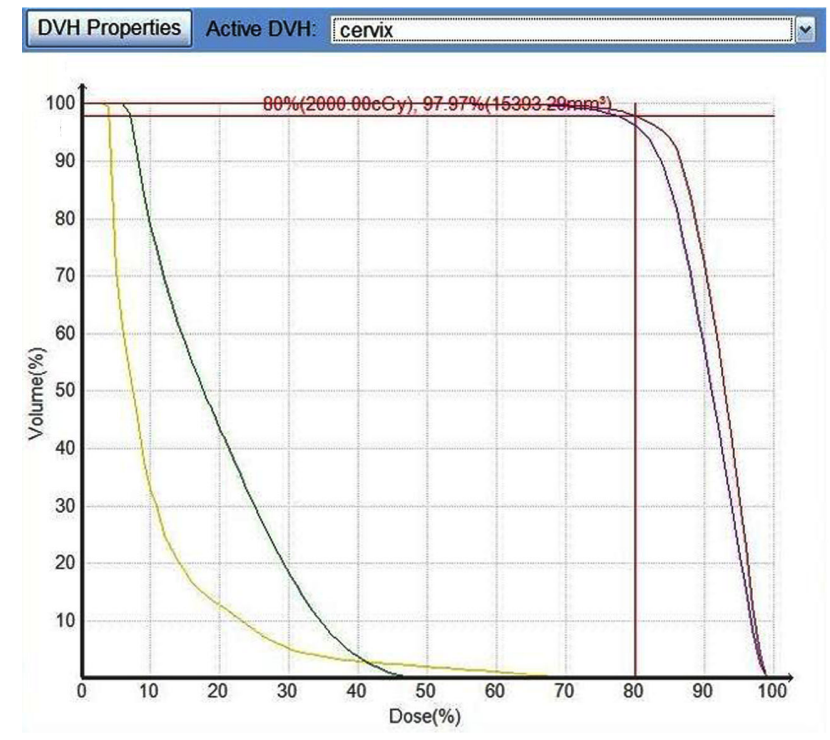

FIGURE 1 | (A) Representative treatment plan for a patient receiving an SBRT boost of $20 \mathrm{~Gy}$ delivered in four fractions. The orange line denotes the planning target volume (PTV). (B) Dose volume histogram showing the bladder (yellow) rectum (green), cervix (red), and PTV (purple).

Other studies using a conventional linear accelerator have reported on a stereotactic boost for gynecologic cancers. In a case study, Hsieh et al. (2010) reported on a patient who was unable to undergo a brachytherapy boost due to multiple uterine myomas. This patient received conventionally fractionated treatment to $54 \mathrm{~Gy}$ followed by a helical tomotherapy boost of $24 \mathrm{~Gy}$ and concurrent chemoradiotherapy. At 14 months there was no evidence of tumor recurrence. She had grade 1 nausea and vomiting during treatment.

Molla et al. (2005) reported on a mixed population of 23 patients with either endometrial $(n=9)$ or cervical $(n=7)$ cancer including two patients with local relapse. The patients received a $14 \mathrm{~Gy}$ boost delivered with a linac-based micromultileaf collimator in two fractions at 4-7 days intervals. At a median 12.6 months follow-up one previously irradiated relapse patient had a grade 
Table 1 | Patient characteristics and treatment summary.

\begin{tabular}{lllllll}
\hline Patient & Stage & Age (years) & $\begin{array}{l}\text { Reason unable to } \\
\text { receive brachytherapy }\end{array}$ & External RT (Gy) & SBRT boost & $\begin{array}{l}\text { SBRT prescription } \\
\text { isodose line }\end{array}$ \\
\hline 1 & IIB & 83 & Anatomy & 50.4 & $6.5 \mathrm{~Gy} \times 3 \mathrm{BED} 77.67 \mathrm{~Gy}$ & 81 \\
2 & IIB & 79 & Medical & 61.2 & $4.0 \mathrm{~Gy} \times 5$ BED $84.93 \mathrm{~Gy}$ & 82 \\
3 & IIB & 75 & Choice & 61.2 & $4.0 \mathrm{~Gy} \times 5$ & 80 \\
4 & IIB & 95 & Medical & 61.2 & $4.0 \mathrm{~Gy} \times 5$ & 81 \\
5 & IV & 71 & Anatomy & 61.2 & $4.0 \mathrm{~Gy} \times 5$ & 80 \\
6 & IVA & 76 & Anatomy & 61.2 & $4.0 \mathrm{~Gy} \times 5$ & 78 \\
\hline
\end{tabular}

Table 2 | Summary of maximum rectal and bladder doses.

\begin{tabular}{lll}
\hline Patient & Rectal max dose (Gy) & Bladder max dose (Gy) \\
\hline 1 & 55.8 & 55.8 \\
2 & 73.4 & 74.2 \\
3 & 68.7 & 70.7 \\
4 & 71.6 & 70.1 \\
5 & 70.7 & 68.7 \\
6 & 67.5 & 73.7 \\
\hline
\end{tabular}

3 rectal bleed; no other patients developed severe urinary or intestinal toxicity. One recurrence occurred 12 months following treatment for a cervical cancer patient; no other failures occurred. Following a treatment planning comparison the authors concluded that SBRT improved dose homogeneity to the PTV and reduced rectal dose compared to brachytherapy. In a follow-up publication, Jorcano et al. (2010) reported on 17 endometrial and 9 cervical cancer patients treated with 45-50.4 Gy EBRT followed by a SBRT boost of $14 \mathrm{~Gy}$ delivered in two fractions. Acute toxicities consisted of 23 and 25\% RTOG grade 3 or less urinary and lower-gastrointestinal toxicities, respectively. At a median 47 months follow-up, the 3-year locoregional failure rate was $96 \%$ for both endometrial and cervical patients. The authors conclude that SBRT is feasible, well tolerated, and could be considered an acceptable alternative to brachytherapy.

Limited reports on robotically delivered SBRT boost for cervical cancer patients have been published. The University of North Carolina reported on treatment with a CyberKnife SBRT boost of $25 \mathrm{~Gy}$ in five fractions for a cervical cancer patient unable to

\section{REFERENCES}

Barraclough, L. H., Swindell, R., Livsey, J. E., Hunter, R. D., and Davidson, S. E. (2008). External beam boost for cancer of the cervix uteri when intracavitary therapy cannot be performed. Int. J. Radiat. Oncol. Biol. Phys. 71, 772-778.

Eifel, P. J., Winter, K., Morris, M., Levenback, C., Grigsby, P. W., Cooper, J., Rotman, M., Gershenson, D., and Mutch, D. G. (2004). Pelvic irradiation with concurrent chemotherapy versus pelvic and para-aortic irradiation for high-risk cervical cancer: an update of radiation therapy oncology group trial (RTOG) 90-01. J. Clin. Oncol. 22, 872-880.

Fletcher, G. H., and Hamberger, A. D. (1980). "Squamous cell carcinoma of the uterine cervix," in Textbook of Radiation Therapy, 3rd Edn, ed. G. H. Fletcher (Philadelphia, PA: Lea and Febiger), 72-778.

Fuller, D. B., Naitoh, J., Lee, C., Hardy, S., and Jin, H. (2008). Virtual HDR CyberKnife treatment for localized prostatic carcinoma: dosimetry comparison with HDR brachytherapy and preliminary undergo brachytherapy. At a follow-up of 10 months the patient exhibited no RTOG toxicities, however, the patient died from progression of liver metastases. In the present series, one patient received 50.4 Gy with a 19.5 Gy SBRT boost and the later five patients received 60 Gy with 20 Gy SBRT boosts delivered using robotic SBRT with real-time motion tracking. The inclusion of motion tracking with CyberKnife delivered SBRT offers a more accurate dose delivery to the target potentially accounting for the lack of significant toxicity in this patient population despite a higher delivered dose than the conventional linear accelerator delivered SBRT boost results (Molla et al., 2005; Jorcano et al., 2010). In addition, the lack of any failure for the five patients with a minimum of 12 months follow-up is highly promising compared to the EBRT boost results for which the cancer-specific overall survival at 1 year was already only $80 \%$ (Barraclough et al., 2008).

\section{CONCLUSION}

This paper is the among the first to report on using robotic SBRT in patients with real-time motion tracking for the treatment of locally advanced cervical cancer in patients who are unable to undergo brachytherapy. These preliminary results suggest that CyberKnife robotic SBRT is a safe and effective modality in the treatment of cervix cancer for those patients unable to undergo brachytherapy. Additional confirmatory prospective studies with larger numbers of patients and longer follow-up are required to validate the durability of these results.

\section{ACKNOWLEDGMENTS}

The authors gratefully acknowledge the editorial assistance of Pam Commike, Ph.D, Accuray Incorporated. clinical observations. Int. J. Radiat. Oncol. Biol. Phys. 70, 1588-1597.

Fyles, A., Keane, T. J., Barton, M., and Simm, J. (1992). The effect of treatment duration in the local control of cervix cancer. Radiother. Oncol. 25, 273-279.

Girinsky, T., Rey, A., Roche, B., Haie, C., Gerbaulet, A., Randrianarivello, H., and Chassagne, D. (1993). Overall treatment time in advanced cervical carcinomas: a critical parameter in treatment outcome. Int J. Radiat. Oncol. Biol. Phys. 27, 1051-1056.
Haripotepornkul, N. H., Nath, S. K., Scanderbeg, D., Saenz, C., and Yashar, C. M. (2011). Evaluation of intra- and inter-fraction movement of the cervix during intensity modulated radiation therapy. Radiother. Oncol. 98, 347-351.

Hombaiah, U., Blake, P., and Bidmead, M. (2006). Movement of the cervix in after-loading brachytherapy: implications for designing external-beam radiotherapy boost fields. Clin. Oncol. (R. Coll. Radiol.) 18, 313-319. 
Hsieh, C. H., Wei, M. C., Hsu, Y. P., Chong, N. S., Chen, Y. J., Hsiao, S. M., Hsieh, Y. P., Wang, L. Y., and Shueng, P. W. (2010). Should helical tomotherapy replace brachytherapy for cervical cancer? Case report. BMC Cancer 10, 637. doi:10.1186/1471-2407-10-637

Jorcano, S., Molla, M., Escude, L., Sanz, S., Hidalgo, A., Toscas, J. I., Linero, D., and Miralbell, R. (2010). Hypofractionated extracranial stereotactic radiotherapy boost for gynecologic tumors: a promising alternative to high-dose rate brachytherapy. Technol. Cancer Res. Treat. 9, 509-514.

Kilby, W., Dooley, J. R., Kuduvalli, G., Sayeh, S., and Maurer, C. R. Jr. (2010). The CyberKnife robotic radiosurgery system in 2010. Technol. Cancer Res. Treat. 9, 433-452.

Lanciano, R. M., Pajak, T. F., Martz, K., and Hanks, G. E. (1993). The influence of treatment time on outcome for squamous cell cancer of the uterine cervix treated with radiation: a patterns-of-care study. Int. J. Radiat. Oncol. Biol. Phys. 25, 391-397.

Molla, M., Escude, L., Nouet, P., Popowski, Y., Hidalgo, A., Rouzaud, M., Linero, D., and Miralbell, R. (2005). Fractionated stereotactic radiotherapy boost for gynecologic tumors: an alternative to brachytherapy? Int. J. Radiat. Oncol. Biol. Phys. 62, 118-124.

Perez, C. A., Grigsby, P. W., Castro-Vita, H., and Lockett, M. A. (1995). Carcinoma of the uterine cervix. I. Impact of prolongation of overall treatment time and timing of brachytherapy on outcome of radiation therapy. Int. J. Radiat. Oncol. Biol. Phys. 32, 1275-1288.

Petereit, D. G., Sarkaria, J. N., Chappell, R., Fowler, J. F., Hartmann, T.
J., Kinsella, T. J., Stitt, J. A., Thomadsen, B. R., and Buchler, D. A. (1995). The adverse effect of treatment prolongation in cervical carcinoma. Int. J. Radiat. Oncol. Biol. Phys. 32, 1301-1307.

Rose, P. G., Bundy, B. N., Watkins, E. B., Thigpen, J. T., Deppe, G., Maiman, M. A., Clarke-Pearson, D. L., and Insalaco, S. (1999). Concurrent cisplatin-based radiotherapy and chemotherapy for locally advanced cervical cancer. N. Engl. J. Med. 340 , 1144-1153.

Taylor, A., and Powell, M. E. (2008). An assessment of interfractional uterine and cervical motion: implications for radiotherapy target volume definition in gynaecological cancer. Radiother. Oncol. 88, 250-257.

Conflict of Interest Statement: Dr. Haas has received speaker's honoraria from Accuray Inc., Sunnyvale, CA, USA.

Received: 18 November 2011; accepted: 20 February 2012; published online: 21 March 2012.

Citation: Haas JA, Witten MR, Clancey $O$, Episcopia K, Accordino D and Chalas E (2012) CyberKnife boost for patients with cervical cancer unable to undergo brachytherapy. Front. Oncol. 2:25. doi: 10.3389/fonc.2012.00025

This article was submitted to Frontiers in Radiation Oncology, a specialty of Frontiers in Oncology.

Copyright $\odot 2012$ Haas, Witten, Clancey, Episcopia, Accordino and Chalas. This is an open-access article distributed under the terms of the Creative Commons Attribution Non Commercial License, which permits non-commercial use, distribution, and reproduction in other forums, provided the original authors and source are credited. 